Pacific

Journal of

Mathematics

REGULARITY OF THE HEAT OPERATOR ON A MANIFOLD WITH CYLINDRICAL ENDS

Thalia D. Jeffres and Paul Loya

Volume $215 \quad$ No. 2

June 2004 


\title{
REGULARITY OF THE HEAT OPERATOR ON A MANIFOLD WITH CYLINDRICAL ENDS
}

\author{
Thalia D. Jeffres and Paul Loya
}

\begin{abstract}
We study mapping properties of the heat operator $e^{t A}$ of an $\boldsymbol{m}$-th order elliptic $b$-differential operator in appropriately defined spaces of whole and fractional (Hölder) derivatives. An application is made to short time existence of solutions to certain semilinear parabolic equations.
\end{abstract}

\section{Introduction}

Let $(M, g)$ be a compact manifold with boundary. We say that $M$ has a cylindrical end if there is a neighborhood in which there is a local diffeomorphism to a product $[0, \varepsilon)_{x} \times N_{z}$ in which the metric may be approximated by

$$
\left(\frac{d x}{x}\right)^{2}+h_{N},
$$

where $h_{N}$ is a Riemannian metric on the cross section $N$. Note that in this metric, the boundary is infinitely far away. $M$ is called a manifold with cylindrical ends if it has at least one cylindrical end and no other type of boundary.

The purpose of this paper is to study differentiability properties of solutions to the heat equation

$$
\left\{\begin{aligned}
\frac{\partial u}{\partial t}-\Delta u & =0 \\
\left.u\right|_{t=0} & =f
\end{aligned}\right.
$$

on such a manifold $M$. The Laplacian in such a metric $g$ will be a $b$ differential operator, that is to say, an operator composed of vector fields tangent to the boundary. In the local coördinates given above, the set of such vector fields is generated by

$$
\left\{x \frac{\partial}{\partial x}, \frac{\partial}{\partial z_{1}}, \ldots, \frac{\partial}{\partial z_{n-1}}\right\}
$$

These are called the boundary vector fields. In fact, the Laplacian could be replaced by $A$, any elliptic combination of the boundary vector fields. See $[\mathrm{Ma}]$ or $[\mathbf{M e}]$ for more on these elliptic operators. Elliptic $b$-operators are essentially self-adjoint. 
Example 1.1. If $M=[0, \infty)$, the heat equation is

$$
\frac{\partial u}{\partial t}-\left(x \frac{\partial}{\partial x}\right)^{2} u=0 .
$$

The relevant analytic feature of this operator is the vanishing coefficient $x$ in the elliptic part, which prevents it from being uniformly elliptic in the traditional sense. It was for the purpose of studying such operators that Melrose developed the $b$-calculus; see his book [Me] and also the paper of Mazzeo [Ma] and the many others referenced in their work. This is exactly what is needed here, for in this point of view the vector field $x \partial / \partial x$ is seen as one indivisible entity rather than $x$ being the coefficient. All spaces and operators are in terms of derivatives by the boundary vector fields. We define $C_{b}^{k}(M)$ to be the space of continuous functions with up to $k$ derivatives by $x \partial / \partial x$ and $\partial / \partial z_{i}$ bounded. We also make use of the Hölder space $C_{b}^{\gamma}(M)$ consisting of continuous functions for which

$$
\|u\|_{\gamma}=\sup _{M}|u|+\sup _{p \neq q} \frac{|u(p)-u(q)|}{\left(d_{g}(p, q)\right)^{\gamma}}<\infty,
$$

where $d_{g}(p, q)$ represents the distance between the points $p$ and $q$ in the cylindrical end metric as long as $p$ and $q$ are 'close'. For a precise definition, see Section 4.

The main theorem is:

Theorem 1.2. Let $e^{t A}$ be the heat operator of an $m$-th order b-differential operator $A$ which is elliptic with respect to a sector whose complement is negative (see Theorem 2.4). Then for any $\alpha \in \mathbb{R}, e^{t A}$ is bounded for $t$ within any fixed bounded interval of $(0, \infty)$, between the following weighted spaces and with the corresponding estimates:

$$
e^{t A}: x^{\alpha} C^{0}(M) \longrightarrow x^{\alpha} C_{b}^{k}(M) ;\|u\|_{k} \leq C t^{-k / m}\|f\|_{0},
$$

and

$$
e^{t A}: x^{\alpha} C_{b}^{\gamma}(M) \longrightarrow x^{\alpha} C_{b}^{m}(M) ;\|u\|_{m} \leq C t^{-1+\gamma / m}\|f\|_{\gamma} .
$$

We note two immediate features of these estimates. One is that the estimates blow up as $t$ approaches zero. This occurs because of the well-known smoothing phenomenon associated to heat transfer. The second observation is that although our spaces are defined in a way that accounts for the singular metric, the powers of $t$ are the same as in the smooth case.

The method of proof is roughly the same also. The solution to the heat equation may be expressed as the integral of the product of the heat kernel with the initial data. For the heat equation of the usual Laplacian in $\mathbb{R}^{n}$, this solution is

$$
u(x, t)=\int_{\mathbb{R}^{n}} \frac{1}{(4 \pi t)^{n / 2}} e^{-\|x-y\|^{2} / 4 t} f(y) d y .
$$


To estimate derivatives, one passes the derivative inside the integral and checks that the integrals converge. One may calculate directly that each successive differentiation produces a factor of $t^{-1 / 2}$. See for example a standard text in PDE such as that of Evans $[\mathbf{E}]$ or Folland $[\mathbf{F}]$. On a smooth compact manifold, or in the interior of our manifold with cylindrical ends, the heat kernel of the metric Laplacian is approximated by that of $\mathbb{R}^{n}$, as one discovers in Aubin $[\mathbf{A}]$. Following a similar method, we explain in Section Two the form of the heat kernel on a manifold with cylindrical ends, and in Sections Three and Four we carry out the calculations.

Boundedness properties of the heat operator $e^{t A}$ in appropriately defined $L^{p}$ and Sobolev spaces have been studied extensively by several authors; see for example $[\mathbf{M a}],[\mathbf{M e}]$, and $[\mathbf{L}]$. Our purpose in investigating the differentiability and Hölder properties is to allow applications to nonlinear problems. An existence theorem for certain semilinear equations will be given in the last section.

This paper is continuation of our earlier paper $[\mathbf{J L}]$ in which we studied the heat equation on a cone manifold, and further description of related work of other authors may be found there.

Finally, we thank the referee for helpful comments in improving the paper.

\section{The heat space and heat kernel}

We wish to express the heat operator as an integral operator, and a detailed understanding of the singular structure of the integral kernel will enable us to determine the mapping properties. Such a description is given in $[\mathbf{L}]$ and we will quote that result in this section after establishing some concepts and notations.

A central idea of Melrose is that when $M$ is singular or has boundary, these integral kernels are more naturally described as functions or distributions not on $M \times M \times[0, \infty)_{t}$, but rather on a more complicated manifold with corners, whose interior is identified with the interior of $M \times M \times[0, \infty)_{t}$. The singularities of the kernel are then described by the asymptotic behavior of the kernel on approach to the various corners, edges, and faces. It is explained in Melrose' book [Me] that the correct space is obtained by an ordinary blow up of $Z \times[0, \infty) \subset M^{2} \times[0, \infty)$, where $Z=(\partial M)^{2} \subset M^{2}$ is the corner, followed by what he calls a parabolic blow up at the diagonal at $t=0$. This space is denoted here by $M_{H}^{2}$ and is a well-defined object with a differentiable structure and so on; all these details may be found in $[\mathbf{M e}]$. We remark that Melrose focused primarily on Laplace-type operators. We wish to include $m$-th order operators. Because the blow up space is not essentially different, we will call this last step of its construction an " $m$-th order parabolic blow up," and we will also use the same notation, $M_{H}^{2}$, to denote it. 
For our immediate purpose here, we only need to know that locally these blow ups are simply introduction of singular coördinates, and these coördinates are very natural because they reflect the familiar radial symmetry of $b$-differential operators and the parabolic symmetry of the heat operator. As a manifold with corners, there is no global coördinate chart for all of $M_{H}^{2}$. We now describe the local charts.

Again let $\left(x, z_{1}, \ldots, z_{n-1}\right)$ be coördinates in a neighborhood of the boundary where $M$ is locally diffeomorphic to $[0, \varepsilon)_{x} \times N_{z}$. Let $\left(y, z_{1}^{\prime}, \ldots, z_{n-1}^{\prime}\right)$ be local coördinates for the second copy of $M$ in $M^{2} \times[0, \infty)$. To compress notation we will often write $z=\left(z_{1}, \ldots, z_{n-1}\right)$ and $z^{\prime}=\left(z_{1}^{\prime}, \ldots, z_{n-1}^{\prime}\right)$. The first blow up is accomplished by the introduction of projective (really polar, reflecting the radial symmetry of the elliptic part — see [G]) coördinates near $Z \times[0, \infty) \subset M^{2} \times[0, \infty)$. At this stage we have two neighborhoods.

\section{Near the left-hand corner}

$$
\left\{t=t, \xi=x / y, \eta=y, z=z, z^{\prime}=z^{\prime}\right\} .
$$

Three boundary hypersurfaces meet at the left-hand corner. Using notation consistent with $[\mathbf{L}]$, we define them as follows:

Definition 2.1. The left boundary, $l b$, is the lift of $\{x=0\}$ and is given in these coördinates by $\{\xi=0\}$. The front face, $f f$, is the blow up of the corner and is given by $\{\eta=0\}$. The temporal boundary, $t b$, is the lift of $\{t=0\}$ and is described in by $\{t=0\}$. This submanifold is also denoted by $M_{b}^{2}$ and is often referred to as the ' $b$-stretched product'.

\section{Near the right-hand corner}

$$
\left\{t=t, \xi^{\prime}=x, \eta^{\prime}=y / x, z=z, z^{\prime}=z^{\prime}\right\} .
$$

Definition 2.2. The right boundary, $r b$, is given by $\left\{\eta^{\prime}=0\right\}$.

Note however that approach to the right boundary can also be described in the coördinates $\mathbf{I}$. by $\xi \rightarrow \infty$.

Next we do the $m$-th order parabolic blow up of the lifted diagonal $\{x=$ $\left.y, z=z^{\prime}\right\} \cap\{t=0\}$. This set belongs to either coördinate patch, so choosing those near the left-hand corner, it is described locally by $\left\{\xi=1, z=z^{\prime}\right\} \cap$ $\{t=0\}$. Parabolic blow up is introduction of a singular system of coördinates in which three data are used to locate a point: Its position $\eta$ along the diagonal, its location $\left(\omega_{0}, \omega^{\prime}\right)$ on a unit reference heat sphere $\mathbb{S}_{H}^{n}$ centered at $\left(\xi, z-z^{\prime}, \eta, t\right)=(1,0, \eta, 0)$, and its parabolic distance from the center. The upper hemisphere of the unit heat sphere with center at the origin is defined to be

$$
\mathbb{S}_{H}^{n}=\left\{\vec{\omega}=\left.\left(\omega_{0}, \omega^{\prime}\right) \in \mathbb{R}^{n+1}\left|\omega_{0} \geq 0, \omega_{0}^{2}+\right| \omega^{\prime}\right|^{2 m}=1\right\},
$$


and the parabolic distance of $\left(\xi, z-z^{\prime}, \eta, t\right)$ to $(1,0, \eta, 0)$ is

$$
d=\sqrt[2 m]{t^{2}+\left|\left(\xi-1, z-z^{\prime}\right)\right|^{2 m}} .
$$

Global "coördinates" are

$$
\left\{\omega_{0}=t / d^{m}, \omega^{\prime}=(\xi-1) / d,\left(z-z^{\prime}\right) / d, \eta, z^{\prime}, d\right\} .
$$

There appears to be an extra coördinate because $\omega_{0}$ and $\omega^{\prime}$ are not independent. In the regions where $\xi-1$ and $z-z^{\prime}$ are small compared to $t$, i.e., near the top of the blown-up diagonal, local coördinates are

$$
\left\{\rho=t^{1 / m}, w=\frac{\xi-1}{t^{1 / m}}=\frac{x-y}{y t^{1 / m}}, \zeta=\frac{z-z^{\prime}}{t^{1 / m}}, \eta=y, z^{\prime}=z^{\prime}\right\} .
$$

Near the top where the blown-up diagonal meets the front face, there are two boundary hypersurfaces, the front face and a new one.

Definition 2.3. The blown-up diagonal is called the temporal face and is denoted by $t f$. It is locally given by $\{\rho=0\}$.

We could also define a separate coördinate system valid near $t b$ where the diagonal hits the front face, but it won't be necessary because $t b$ is reached as $|(w, \zeta)| \rightarrow \infty$.

We now recall an ellipticity condition that guarantees the existence of the heat operator for a $b$-differential operator. Let $A$ be an $m$-th order $b$ differential operator on $M$. Then on a neighborhood $[0, \varepsilon)_{x} \times N$ near the boundary, we can write

$$
A=\sum_{k=0}^{m} A_{m-k}(x) \cdot\left(x \frac{\partial}{\partial x}\right)^{k}
$$

where the $A_{m-k}$ 's are differential operators of order $m-k$ on $N$ depending smoothly in $x$. The $b$-principal symbol of $A, a_{b}$, is just the usual principal symbol of $A$ in the interior of $M$, and in the decomposition above is defined by

$$
a_{b}(\xi)=\sum_{k=0}^{m} a_{m-k}\left(x, \xi^{\prime}\right) \cdot\left(i \xi_{1}\right)^{k}, \quad \xi=\left(\xi_{1}, \xi^{\prime}\right) \in \mathbb{R} \times T^{*}(N),
$$

where $a_{m-k}\left(x, \xi^{\prime}\right)$ is the principal symbol of the operator $A_{m-k}(x)$ as a differential operator on $N$. The $b$-principal symbol $a_{b}$ is a function on the $b$-cotangent bundle (see $[\mathbf{M e}]) . A$ is said to be elliptic with respect to a sector $\Lambda \subset \mathbb{C}$ if $a_{b}(\xi)-\lambda \neq 0$ for all $\lambda \in \Lambda \cup\{0\}$ and $b$-cotangent vectors $\xi \neq 0$. Finally, a sector $\Lambda$ is said to be negative if it is of the form $\Lambda=\{\lambda \in$ $\mathbb{C} \mid \pi-\delta<\arg \lambda<\pi+\delta\}$ for some $0<\delta<\pi / 2$.

For instance, the principal symbol of the one-dimensional Laplacian $\Delta=$ $\left(x \partial_{x}\right)^{2}$ is $-\xi^{2}$. Since $-\xi^{2}-\lambda \neq 0$ for $\xi \neq 0$ and for $\lambda$ not on the negative 
real axis, $\Delta$ is elliptic with respect to any sector whose complement is negative. In general, the Laplacian on a manifold with cylindrical ends is always elliptic with respect to any sector whose complement is negative.

Theorem $2.4([\mathbf{L}])$. Let $A$ be an $m$-th order b-differential operator that is elliptic with respect to a sector whose complement is negative. Then the heat operator $e^{t A}$ exists and its Schwartz kernel, when written in the singular coördinates of $M_{H}^{2}$, defines a smooth function on the interior of $M_{H}^{2}$ that vanishes to infinite order at $l b, r b$, and $t b$, is smooth up to ff, and finally, has an expansion up to tf with leading power given by $-n$.

The primary example of an operator satisfying the conditions of this theorem is the Laplacian with respect to a cylindrical end metric (cf. Theorem 7.24 of $[\mathrm{Me}])$.

In Theorem 2.4, we choose to treat the kernel $\kappa_{H}$ of the heat operator $H=e^{t A}$ as a function. In other words, the action of the operator on the initial data $f$, also a function, will be to multiply $f$ by $\kappa_{H}$ and integrate against the Riemannian volume form:

$$
H f(x, z, t)=\int \kappa_{H}\left(x, y, z, z^{\prime}, t\right) f\left(y, z^{\prime}\right) y^{-1} d y d z^{\prime} .
$$

To illustrate the above theorem, consider the coördinates $\rho=t^{1 / m}, w=$ $(\xi-1) / t^{1 / m}=(x-y) / y t^{1 / m}, \zeta=\left(z-z^{\prime}\right) / t^{1 / m}, \eta=y$, and $z^{\prime}=z^{\prime}$ near the blown-up diagonal. Here, $\rho$ defines $t f, \eta$ defines $f f$, and $t b$ corresponds to the face in the limit $|(w, \zeta)| \rightarrow \infty$. Then the above theorem states that we can write

$$
\kappa_{H}=H\left(\rho, w, \zeta, \eta, z^{\prime}\right),
$$

where $H$ is a function that vanishes to infinite order as $|(w, \zeta)| \rightarrow \infty$, is smooth in $\eta$ and $z^{\prime}$, and finally, has an expansion as $\rho \rightarrow 0$ with leading power $-n$. Thus, we have

$$
H \sim \sum_{j=0}^{\infty} \rho^{-n+j} a_{j}\left(w, \zeta, \eta, z^{\prime}\right) \text { as } \rho \rightarrow 0,
$$

where the $a_{j}$ 's are smooth functions vanishing to infinite order as $|(w, \zeta)| \rightarrow$ $\infty$ and smooth in $\eta$ and $z^{\prime}$.

\section{Local calculations of sup and whole derivative bounds}

This section and the next comprise the proof of Theorem 1.2.

Definition 3.1. The space $C_{b}^{k}(M)$ consists of continuous functions on $M$ such that

$$
\sup |u|+\sup \left|V_{1} u\right|+\cdots+\sup \left|V_{1} \cdots V_{k} u\right|<\infty
$$

for every choice of $k$ boundary vector fields. 
Recall that the boundary vector fields are locally generated by vector fields $x \partial / \partial x$ and $\partial / \partial z_{i}$.

Henceforth, we fix an $m$-th order $b$-differential operator $A$ that is elliptic with respect to a sector whose complement is negative. We begin by reducing Theorem 1.2 to the case of zero weights. Indeed, observe that for any $\alpha \in \mathbb{R}$, we have

$$
e^{t A}: x^{\alpha} C^{0}(M) \longrightarrow x^{\alpha} C_{b}^{k}(M) \Longleftrightarrow x^{-\alpha} e^{t A} x^{\alpha}: C^{0}(M) \longrightarrow C_{b}^{k}(M) .
$$

If $\kappa_{H}$ denotes the Schwartz kernel of $e^{t A}$, then $x^{-\alpha} \kappa_{H} y^{\alpha}=\xi^{-\alpha} \kappa_{H}$ is the kernel of $x^{-\alpha} e^{t A} x^{\alpha}$. By Theorem 2.4, $\kappa_{H}$ vanishes to infinite order at $l b=$ $\{\xi=0\}$ and $r b=\{\xi=\infty\}$. Since the multiplier $\xi^{-\alpha}$ can only affect the asymptotics of $\kappa_{H}$ at the left and right boundaries, it follows that the kernels of $e^{t A}$ and $x^{-\alpha} e^{t A} x^{\alpha}$ have the same asymptotics as functions on $M_{H}^{2}$. We prove Theorem 1.2 using only the asymptotic properties of the heat kernel, and thus without loss of generality we assume that $\alpha=0$. We examine the heat kernel in each of the coördinate patches on $M_{H}^{2}$ separately, multiplying by a cut-off function so that we can assume each time that the heat kernel is supported in a box within that coördinate patch.

\section{Estimates near the left and right-hand corners}

Recalling the local coördinates from Section Two, we write the heat operator out, for simplicity omitting the $z$ and $z^{\prime}$ variables corresponding to the compact cross section and which play no role yet. Here $H$ stands for both the operator and its integral kernel:

$$
H f(x, t)=\int H\left(t, \frac{x}{y}, y\right) f(y) y^{-1} d y .
$$

According to Theorem 2.1 above, $H(t, \xi, y)$ vanishes to infinite order as $t \rightarrow 0$ or as $\xi=x / y \rightarrow 0 ; H$ is also vanishing to infinite order as $\xi \rightarrow \infty$, for this is approach to the right boundary. We use this infinite vanishing to absorb the nonintegrable factor of $y^{-1}$ appearing in the measure, by introducing a change of variables, $u=y / x$. Then

$$
H f(x, t)=\int H\left(t, \frac{1}{u}, x u\right) f(x u) u^{-1} d u .
$$

Absorbing $u^{-1}$ into a new kernel by defining

$$
G\left(t, \frac{1}{u}, x u\right)=u^{-1} H\left(t, \frac{1}{u}, x u\right),
$$

the new kernel $G$ is still vanishing to infinite order at both ends, $u \rightarrow 0$ and $u \rightarrow \infty$. Therefore,

$$
H f(x, t)=\int G\left(t, \frac{1}{u}, x u\right) f(x u) d u
$$


and estimating $f$ by its maximum,

$$
|H f(x, t)| \leq C\|f\|_{0} .
$$

For the derivatives, we pass the derivative inside the integral,

$$
\begin{aligned}
x \frac{\partial}{\partial x}(H f)(x, t) & =\int x \frac{\partial H}{\partial \xi} \frac{\partial \xi}{\partial x} f(y) y^{-1} d y \\
& =\int x \frac{\partial H}{\partial \xi} \frac{1}{y} f(y) y^{-1} d y \\
& =\int \xi \frac{\partial H}{\partial \xi} f(y) y^{-1} d y .
\end{aligned}
$$

Now note that because the kernel $H$ was vanishing to infinite order in $\xi$ at both zero and infinity, the very same is true of $\xi \partial H / \partial \xi$. In fact, any finite number of derivatives can be applied and the resulting factors of $\xi^{k}$ can be absorbed without changing the nature of the asymptotics. Therefore, we find ourselves with a kernel of the same description as $H$ itself, and may apply the same argument as used in the sup estimate. There is never any problem with the $z_{i}$ derivatives, since these represent compact directions, so we obtain

$$
\left|\left(x \frac{\partial}{\partial x}\right)^{k} \frac{\partial}{\partial z_{i_{1}}} \ldots \frac{\partial}{\partial z_{i_{l}}} H f(x, z, t)\right| \leq C_{k+l}\|f\|_{0} .
$$

\section{Where the diagonal hits the front face}

For the first time the coördinates in the direction of the cross section appear, and we write the action of the heat operator on $f$ in these local coördinates as

$$
H f(x, z, t)=\int H\left(t^{1 / m}, \frac{x-y}{y t^{1 / m}}, \frac{z-z^{\prime}}{t^{1 / m}}, y, z^{\prime}\right) f\left(y, z^{\prime}\right) y^{-1} d y d z^{\prime},
$$

with leading asymptotic $H \sim \rho^{-n}=t^{-n / m}$ and vanishing to infinite order as $|(w, \zeta)| \rightarrow \infty$. Upon first inspection it looks as though the sup bound will produce an unexpectedly bad factor of $t^{-n / m}$, but once again we can use the variables in which $H$ vanishes to infinite order to absorb some of this. Letting $w$ and $\zeta$ be the variables of integration, we have

$$
\begin{aligned}
H f(x, z, t)=\int & H\left(t^{1 / m}, w, \zeta, y, z^{\prime}\right) f\left(\frac{x}{w t^{1 / m}+1}, z-t^{1 / m} \zeta\right) \\
& \cdot \frac{t^{1 / m}}{w t^{1 / m}+1} t^{(n-1) / m} d w d \zeta .
\end{aligned}
$$


The total factor of $t$ coming from the measure is now $t^{+n / m}$ and we cancel this with the $t^{-n / m}$ in the kernel and call the new kernel $G$ to get

$$
\begin{aligned}
H f(x, z, t)= & \int G\left(t^{1 / m}, w, \zeta, y, z^{\prime}\right) f\left(\frac{x}{w t^{1 / m}+1}, z-t^{-1 / m} \zeta\right) \\
& \cdot \frac{1}{w t^{1 / m}+1} d w d \zeta .
\end{aligned}
$$

Under the assumption that the heat kernel is supported in a small box around the diagonal, the factor $1 /\left(w t^{1 / m}+1\right)$ may be bounded above, because the denominator equals $\xi$, which is one at the diagonal. The new kernel $G$ is bounded in $\rho=t^{1 / m}, y$, and $z^{\prime}$, plus is rapidly decreasing in $w$ and $\zeta$, so that

$$
|H f(x, z, t)| \leq C\|f\|_{0} .
$$

For the derivative in the transversal direction, we calculate

$$
x \frac{\partial}{\partial x}(H f)(x, z, t)=\int\left(w \frac{\partial H}{\partial w}+t^{-1 / m} \frac{\partial H}{\partial w}\right) \cdot f\left(y, z^{\prime}\right) y^{-1} d y d z^{\prime} .
$$

The factor of $t^{-1 / m}$ comes outside the integral, and the remaining terms inside the integral have the same asymptotics as the kernel $H$ itself, so we apply to the integral the same argument as above in the sup bound and obtain the estimate

$$
\left|x \frac{\partial}{\partial x}(H f)(x, z, t)\right| \leq t^{-1 / m} C\|f\|_{0} .
$$

It is easily seen that derivatives by $\partial / \partial z_{i}$ also produce a factor of $t^{-1 / m}$ and do not otherwise change the asymptotics. Each successive differentiation by a boundary vector field produces a new factor of $t^{-1 / m}$ so that in the end we have

$$
\left|\left(x \frac{\partial}{\partial x}\right)^{k} \frac{\partial}{\partial z_{i_{1}}}, \ldots, \frac{\partial}{\partial z_{i_{l}}}(H f)(x, z, t)\right| \leq C_{k+l} t^{-(k+l) / m}\|f\|_{0} .
$$

\section{The Hölder estimates}

We define $d_{g}(p, q)$ as the geometric distance between $p$ and $q$ if this distance is less than or equal to 1 , and 1 if the geometric distance is greater than 1 .

Definition 4.1. For $0<\gamma<1$, the Hölder space $C_{b}^{\gamma}(M)$ contains those functions which are continuous and for which

$$
\|u\|_{\gamma}=\sup _{M}|u|+\sup _{p \neq q} \frac{|u(p)-u(q)|}{\left(d_{g}(p, q)\right)^{\gamma}}<\infty .
$$


There is a simple description of the distance $d_{g}$ in local coördinates. Near the boundary, the cylindrical end metric is of the form

$$
\left(\frac{d x}{x}\right)^{2}+h=d(\log x)^{2}+h,
$$

where $h$ is a metric on $N$. Let $(x, z)$ be coördinates in a neighborhood of the boundary in $M$ and let $\left(y, z^{\prime}\right)$ be a second copy of the same coördinates. In these coördinates, the square of the geometric distance between $(x, z)$ and $\left(y, z^{\prime}\right)$ has the same order of magnitude as

$$
|\log x-\log y|^{2}+\left|z-z^{\prime}\right|^{2}=u^{2}+\left|z-z^{\prime}\right|^{2},
$$

where $u=\log (x / y)$ takes values in $(-\infty, \infty)$. If $v=(x-y) /(x+y)$, then $v$ takes values in $[-1,1]$, and a short computation shows that

$$
v=\frac{1-e^{-u}}{1+e^{-u}} \Longleftrightarrow u=\log \frac{v+1}{1-v} .
$$

Thus, each $u$ corresponds to a unique $v$ and each $v$ corresponds to a unique $u$. Moreover, $u=-\infty$ corresponds to $v=-1, u=0$ corresponds to $v=0$, and $u=+\infty$ corresponds to $v=+1$, and hence the squared distance (2) is has the same order of magnitude as

$$
v^{2}+\left|z-z^{\prime}\right|^{2}=\left(\frac{x-y}{x+y}\right)^{2}+\left|z-z^{\prime}\right|^{2},
$$

as long as $\log (x / y)$ is bounded by some fixed constant.

In view of this discussion, the Hölder part of the norm has the same order of magnitude as

$$
\frac{\left|u(x, z)-u\left(y, z^{\prime}\right)\right|}{|(x-y) /(x+y)|^{\gamma}+\sum_{1}^{n-1}\left|z_{i}-z_{i}^{\prime}\right|^{\gamma}}
$$

which after multiplication by $|x+y|^{\gamma}$ can be written as

$$
\frac{|x+y|^{\gamma}\left|u(x, z)-u\left(y, z^{\prime}\right)\right|}{|x-y|^{\gamma}+|x+y|^{\gamma} \sum_{1}^{n-1}\left|z_{i}-z_{i}^{\prime}\right|^{\gamma}} .
$$

We now derive Hölder estimates on the heat operator. To do so, we apply $m$ derivatives to $H f$ and pass the derivatives inside to get

$$
\begin{aligned}
\left(x \frac{\partial}{\partial x}\right)^{m} H f(x, z, t)= & \int\left(x \frac{\partial}{\partial x}\right)^{m} H \cdot f\left(y, z^{\prime}\right) y^{-1} d y d z^{\prime} \\
= & \int\left(x \frac{\partial}{\partial x}\right)^{m} H \cdot\left[f\left(y, z^{\prime}\right)-f(x, z)\right] y^{-1} d y d z^{\prime} \\
& +f(x, z) \int\left(x \frac{\partial}{\partial x}\right)^{m} H \cdot y^{-1} d y d z^{\prime} .
\end{aligned}
$$


The first integral is estimated using the fact that the initial data function $f$ is bounded in the Hölder norm:

$$
\begin{aligned}
& \left|\int\left(x \frac{\partial}{\partial x}\right)^{m} H \cdot\left[f\left(y, z^{\prime}\right)-f(x, z)\right] y^{-1} d y d z\right| \\
& \leq \int\left|\left(x \frac{\partial}{\partial x}\right)^{m} H\right| \cdot\left|f\left(y, z^{\prime}\right)-f(x, z)\right| y^{-1} d y d z^{\prime} \\
& \leq\|f\|_{\gamma} \int\left|\left(x \frac{\partial}{\partial x}\right)^{m} H\right| \frac{|x-y|^{\gamma}+|x+y|^{\gamma} \sum_{1}^{n-1}\left|z_{i}-z_{i}^{\prime}\right|^{\gamma}}{|x+y|^{\gamma}} y^{-1} d y d z^{\prime} \\
& =\|f\|_{\gamma} \int\left|\left(x \frac{\partial}{\partial x}\right)^{m} H\right| \cdot \frac{|x-y|^{\gamma}}{|x+y|^{\gamma}} y^{-1} d y d z^{\prime} \\
& \quad+\|f\|_{\gamma} \sum_{1}^{n-1} \int\left|\left(x \frac{\partial}{\partial x}\right)^{m} H\right| \cdot\left|z_{i}-z_{i}^{\prime}\right|^{\gamma} y^{-1} d y d z^{\prime} .
\end{aligned}
$$

In the first term, notice that $x$ and $y$ are both positive, so that $|x+y|^{\gamma}>$ $|y|^{\gamma}$, and therefore

$$
\frac{|x-y|^{\gamma}}{|x+y|^{\gamma}} \leq \frac{|x-y|^{\gamma}}{y^{\gamma}}=\frac{|x-y|^{\gamma}}{t^{\gamma / m} y^{\gamma}} t^{\gamma / m}=|w|^{\gamma} t^{\gamma / m} .
$$

In this way, the first term can be rewritten as

$$
\int\left|\left(x \frac{\partial}{\partial x}\right)^{m} H\right| \cdot|w|^{\gamma} t^{\gamma / m} y^{-1} d y d z^{\prime} .
$$

Because $H$ is bounded in compact regions for $w$ and vanishing to infinite order as $|w| \rightarrow \infty$, the $|w|^{\gamma}$ factor can be absorbed into the kernel without changing the asymptotics. The $m$-th derivative of the kernel produces a factor of $t^{-1}$. Therefore, by the sup and whole derivative estimates done above, the first integral can be bounded by $C t^{-1+\gamma / m}\|f\|_{\gamma}$.

Similarly in the second term, in the $i$-th summand, we can write

$$
\left|z_{i}-z_{i}^{\prime}\right|^{\gamma}=\frac{\left|z_{i}-z_{i}^{\prime}\right|^{\gamma}}{t^{\gamma / m}} t^{\gamma / m}=\left|\zeta_{i}\right|^{\gamma} t^{\gamma / m}
$$

and absorb $\left|\zeta_{i}\right|^{\gamma}$ into the kernel without altering its asymptotics. Therefore the second term and also the first integral is bounded in terms of

$$
t^{-1+\gamma / m} \cdot\|f\|_{\gamma}
$$

To estimate the second integral in (3), we show that

$$
\left|\int\left(x \frac{\partial}{\partial x}\right)^{m} H \cdot y^{-1} d y d z^{\prime}\right| \leq C t^{-1+1 / m} \text {. }
$$


This estimate implies that for $t \leq 1$,

$$
\begin{aligned}
\left|f(x, z) \int\left(x \frac{\partial}{\partial x}\right)^{m} H \cdot y^{-1} d y d z^{\prime}\right| & \leq\|f\|_{0} \cdot C t^{-1+1 / m} \\
& \leq C t^{-1+\gamma / m}\|f\|_{\gamma} .
\end{aligned}
$$

Together with the estimate on the first integral in (3) we have

$$
\left|\left(x \frac{\partial}{\partial x}\right)^{m} H f(x, z, t)\right| \leq C t^{-1+\gamma / m}\|f\|_{\gamma} .
$$

Similar arguments give analogous estimates for derivatives by the vector fields $\partial / \partial z_{i}$. This concludes the proof of Theorem 1.2.

To prove the estimate (4), we consider the heat kernel in coördinates where the diagonal hits the front face:

$$
\begin{aligned}
& \int\left(x \frac{\partial}{\partial x}\right)^{m} H \cdot y^{-1} d y d z^{\prime} \\
& =\int\left(x \frac{\partial}{\partial x}\right)^{m} H\left(t^{1 / m}, \frac{\log (x / y)}{t^{1 / m}}, \frac{z-z^{\prime}}{t^{1 / m}}, y, z^{\prime}\right) y^{-1} d y d z^{\prime}
\end{aligned}
$$

where we use $\log (x / y)$ in place of the coördinate $x / y-1$ that we have been using. Here, we used the fact that $\log 1=0$ and the logarithm is a diffeomorphism of $\mathbb{R}^{+}$onto $\mathbb{R}$ to justify this substitution. Setting $v=$ $\log (x / y) / t^{1 / m}$ and $\zeta=\left(z-z^{\prime}\right) / t^{1 / m}$, the kernel $H\left(t^{1 / m}, v, \zeta, y, z^{\prime}\right)$ has the leading asymptotics $H \sim t^{-n / m}$ and vanishes to infinite order as $|(v, \zeta)| \rightarrow$ $\infty$.

Since $y=x e^{-t^{1 / m} v}, z^{\prime}=z-t^{1 / m} \zeta$, and $d y / y d z^{\prime}=t^{n / m} d v d \zeta$, we can write

$$
\begin{aligned}
& \int\left(x \frac{\partial}{\partial x}\right)^{m} H \cdot y^{-1} d y d z^{\prime} \\
& =t^{-1+n / m} \int\left(\frac{\partial^{m} H}{\partial v^{m}}\right)\left(t^{1 / m}, v, \zeta, x e^{-t^{1 / m} v}, z-t^{1 / m} \zeta\right) d v d z^{\prime}
\end{aligned}
$$

where the partial derivatives in $v$ apply only to the second variable in $H\left(t^{1 / m}, v, \zeta, y, z^{\prime}\right)$. With $v=\log (x / y) / t^{1 / m}$ and $\zeta=\left(z-z^{\prime}\right) / t^{1 / m}$, we have

$$
\begin{aligned}
\frac{\partial}{\partial v}\left(\frac{\partial^{m-1} H}{\partial v^{m-1}}\right) & =\left(\frac{\partial^{m} H}{\partial v^{m}}\right)-t^{1 / m} x e^{-t^{1 / m} v}\left(\frac{\partial}{\partial y} \cdot \frac{\partial^{m-1} H}{\partial v^{m-1}}\right) \\
& =\left(\frac{\partial^{m} H}{\partial v^{m}}\right)-t^{1 / m}\left(y \frac{\partial}{\partial y} \cdot \frac{\partial^{m-1} H}{\partial v^{m-1}}\right) .
\end{aligned}
$$

Now integrating by parts and using the fact that $H$ vanishes to infinite order as $|(v, \zeta)| \rightarrow \infty$ shows that Equation (5) is equal to

$$
t^{-1+n / m} \cdot t^{1 / m} \int\left(y \frac{\partial}{\partial y} \cdot \frac{\partial^{m-1} H}{\partial v^{m-1}}\right)\left(t^{1 / m}, v, \zeta, x e^{-t^{1 / m} v}, z-t^{1 / m} \zeta\right) d v d z^{\prime}
$$


where we recall that $y=x e^{-t^{1 / m} v}$. Since $H\left(t^{1 / m}, v, \zeta, y, z^{\prime}\right) \sim t^{-n / m}$ and vanishes to infinite order as $|(v, \zeta)| \rightarrow \infty$, this integral is bounded by a constant times $t^{-1+1 / m}$. The estimate (4) is now proved.

\section{Applications to semilinear problems}

In this section we apply the above results to obtain solutions to some semilinear equations. The reaction-diffusion equations are famous examples of such equations with wide applicability to problems in physics and engineering. Other equations, applications, and theory may be found in the book of Cazenave and Haraux, $[\mathbf{C H}]$, dedicated to this subject. Taylor's volume on nonlinear PDE's, [T], also provides a thorough discussion of nonlinear parabolic equations and includes many interesting physical examples.

The book of Taylor, $[\mathbf{T}]$, explains how to reformulate a nonlinear problem as a contraction mapping. Suppose in addition to the linear part $A u$, our equation also involves quadratic and higher order terms in $u$ and its first derivatives by boundary vector fields. We denote these higher order terms by $Q(u, V u)$, separate them out, and rewrite the equation as

$$
\frac{\partial u}{\partial t}-A u-Q(u, V u)=0, \quad u(0)=f .
$$

Treating $Q$ as an inhomogeneous term and applying Duhamel's principle, Taylor proves the following result:

Theorem 5.1 ([T], p. 273). Suppose that $X$ and $Y$ are Banach spaces such that the following four conditions are satisfied:

1. $e^{t A}: X \longrightarrow X$ is a strongly continuous semigroup, for $t \geq 0$.

2. $Q: X \longrightarrow Y$ is locally Lipschitz.

3. $e^{t A}: Y \longrightarrow X$, for $t>0$.

4. For some $-\gamma>-1$,

$$
\left\|e^{t A}\right\|_{L(Y, X)} \leq C t^{-\gamma}, \quad \text { for } 0<t \leq 1 .
$$

Then the initial value problem (6) with $f \in X$ has a unique solution $u \in$ $C([0, T], X)$ where $T>0$ and is estimable from below in terms of $\|f\|_{X}$.

Interpreting this in the context of a manifold with cylindrical ends, we have the following:

Theorem 5.2. On a manifold with cylindrical ends, let either $Y=C^{0}(M)$ and $X=C_{b}^{m-1}(M)$ or else $Y=C_{b}^{\gamma}(M)$ and $X=C_{b}^{m}(M)$, and let $Q: X \longrightarrow$ $Y$ be locally Lipschitz. Then given any initial data in $X$, the semilinear initial value problem (6) has a solution in $X$ existing on some time interval $[0, T]$ for some $T>0$. 
Proof. The third and fourth conditions are exactly the content of Theorem 1.2. Therefore the only hypothesis that remains to check is the semigroup property. Namely, we need to show that

$$
\|H f(\cdot, t)-f\|_{X} \rightarrow 0 \text { as } t \rightarrow 0 .
$$

This result is very similar to the proof given in Section Six of the earlier paper, $[\mathbf{J L}]$. Let us simply note here that local calculations similar to the ones we gave in Sections Three and Four show that away from the diagonal, the heat operator becomes more like the zero map as $t \rightarrow 0$, while at the diagonal it becomes more and more like the identity operator. For details we refer the reader to $[\mathbf{J L}]$.

As a concluding remark, we observe that long time existence of solutions involves completely different considerations. The reaction-diffusion equation, for example, models a fluid or gas that is diffusing while simultaneously undergoing a chemical reaction. The nonlinear term representing the reaction may be a growth term which is in competition with the linear diffusion term, and which is stronger is highly dependent on the individual problem. Likewise, in geometric applications, global aspects such as curvature and topology influence the long time behavior of solutions.

\section{References}

[A] T. Aubin, Some Nonlinear Problems in Riemannian Geometry, Springer-Verlag, New York, 1998, MR 1636569, Zbl 0896.53003.

[CH] T. Cazenave and A. Haraux, An Introduction to Semilinear Evolution Equations (Translated from the 1990 French original by Yves Martel and revised by the authors), The Clarendon Press, Oxford University Press, New York, 1998, MR 1691574, Zbl 0926.35049.

[E] L.C. Evans, Partial Differential Equations, American Mathematical Society, Providence, 1998, MR 1625845, Zbl 0902.35002.

[F] G. Folland, Introduction to Partial Differential Equations, Princeton University Press, Princeton, 1976, MR 0599578, Zbl 0325.35001.

[G] D. Grieser, Basics of the b-calculus, in 'Approaches to singular analysis' (Berlin, 1999), Birkhäuser, Basel, 2001, 30-84, MR 1827170, Zbl 0987.58011.

[JL] T. Jeffres and P. Loya, Regularity of solutions of the heat equation on a cone, Int. Math. Res. Not., 3 (2003), 161-178, MR 1932532.

[L] P. Loya, Tempered operators and the heat kernel and complex powers of elliptic pseudodifferential operators, Comm. Partial Differential Equations, 26(7-8) (2001), 1253-1321, MR 1855279, Zbl 1008.58019.

[Ma] R. Mazzeo, Elliptic theory of differential edge operators, I, Comm. Partial Differential Equations, 16(10) (1991), 1615-1664, MR 1133743, Zbl 0745.58045.

[Me] R. Melrose, The Atiyah-Patodi-Singer Index Theorem, Research Notes in Mathematics, 4, AK Peters, Wellesley, 1993, MR 1348401, Zbl 0796.58050. 
[T] M. Taylor, Partial Differential Equations, III, Springer-Verlag, New York, 1997, MR 1477408.

Received September 24, 2003. The second author was supported in part by a Ford Foundation fellowship.

UNIVERSIDAD MICHOACANA

MORELIA

MeXico

E-mail address: thalia@ginette.ifm.umich.mx

Department of Mathematics

SUNY AT BINGHAMTON

Binghamton, NY 13902

E-mail address: paul@math.binghamton.edu 